\title{
Hand-finger model of the human heart - a useful educational device for understanding basic position of the cardiac chambers
}

\author{
Lazar Jelev \\ Department of Anatomy, Histology and Embryology, Medical University of Sofia, Sofia, Bulgaria
}

\begin{abstract}
Teaching heart anatomy for the medical students is a difficult task even for the most experienced professors. A practical approach of teaching complicated structure of the cardiac chambers and their space relations is presented here. It is based on a simple and easily understandable hand-finger model of the human heart showing in once the position of the cardiac chambers and the respective heart valves.
\end{abstract}

Keywords: cardiac chambers anatomy; hand-finger model; practical approach

Anatomy 2015;9(2):79-80 @2015 Turkish Society of Anatomy and Clinical Anatomy (TSACA)

\section{Introduction}

The first time when a medical student meets with the real heart during human dissection courses, he or she feels a little bit confused. The preliminary school knowledge about basic heart morphology is no longer enough and the students sink in the ocean of textbooks descriptions and schemes from the atlases, trying to understand the body position of the heart and its chambers. The complicated structure of the cardiac chambers and their space relations are difficult to be presented even in the most detailed written sources. The detailed 3D computer images are another way of learning heart anatomy, but usually the more detailed images we start with, the deeper confusion we fall.

\section{Hand-Finger Model of the Human Heart}

A basic model of the human cardiac chambers, formed by definite posture of both hands (Figures 1a and b), was developed in our Department of Anatomy, Histology and Embryology in Medical University of Sofia, and it is regularly used for explanations of the heart anatomy at the dissection table and also in lectures. This model represents a frontal view of the human heart in the same manner it should be imagined in the patient's chest. To create the model, one should face the palms of both hands with the left thumb crossing in front the right one. This model pro- vides a good explanation of the direction of the blood, running through the left and right halves of the heart (Figure 1a). The left hand corresponds to the right half of the patient's heart, in the same manner the right hand corresponds to the patient's left heart. The whole second to fifth fingers correspond to the atria. The palm, the thenar region and the first finger correspond well to the inlet (palm) and outlet (thenar and thumb) portions of the heart ventricles and to the great vessels starting from them (distal phalanges of the thumb) (Figure 1a). Imaginary circles around the proximal phalanges of the second to fifth fingers correspond to the atrioventricular valves (Figure 1b). The aortic and pulmonary valves in the model correspond to imaginary circles around the proximal phalanges of the right and left thumbs, respectively. Thus, the model shows clearly an important fact about the difference in the basic structure between the right and left ventricles - the clear separation of the inlet and outlet portions of the right ventricle by the supraventricular crest and also the close relation in the left ventricle between the left atrioventricular and the aortic valves.

\section{Discussion}

The student knowledge about the human heart must start from a basic model, simple enough to be readily under- 


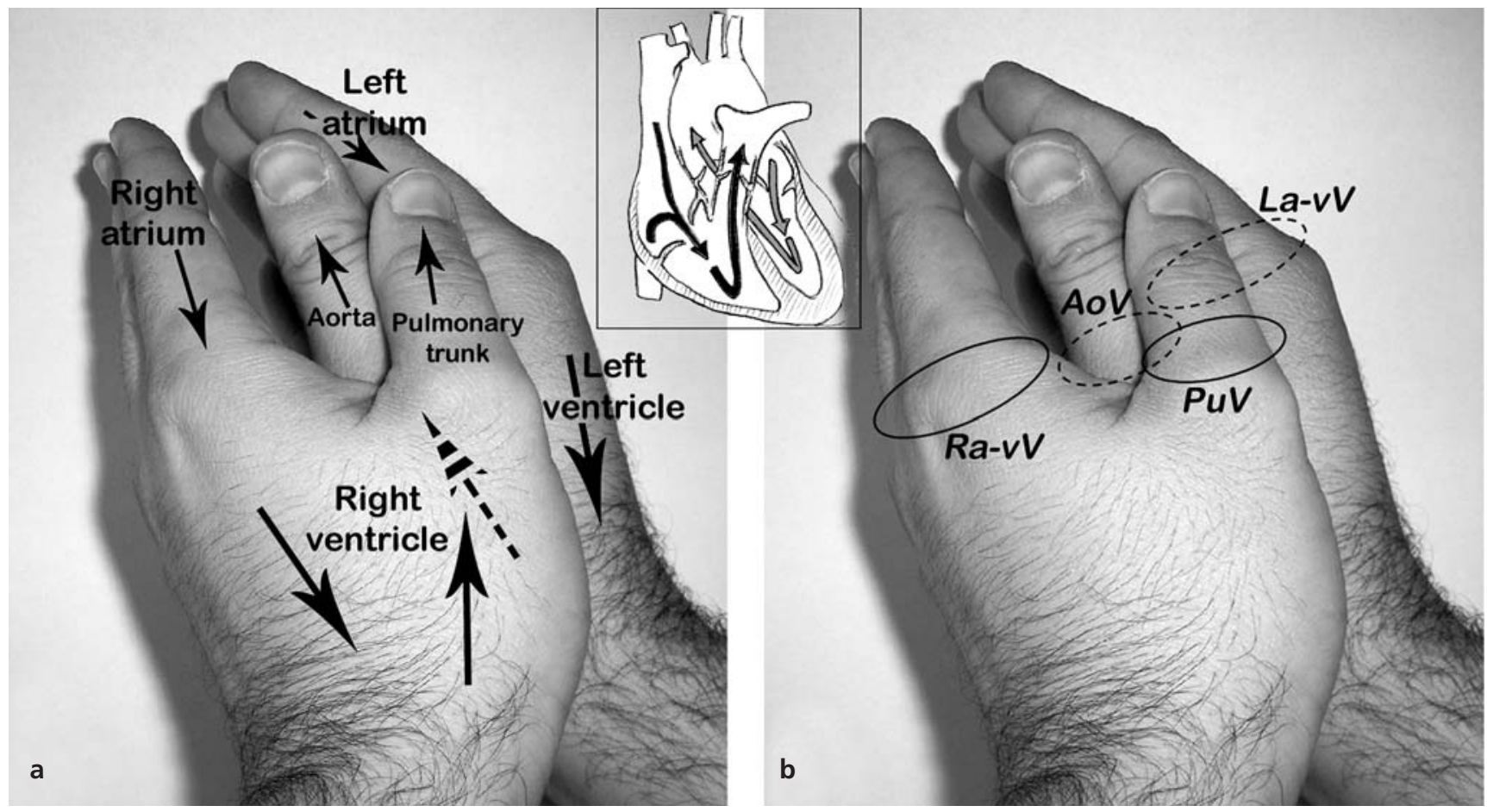

Figure 1. The hand-finger model of the human heart, described in the text, showing the direction of blood running through the right and left heart chambers (a) and the position of the heart valves (b). AoV: aortic valve; La-vV: left atrioventricular valve; PuV: pulmonary valve; Ra-vV: right atrioventrucular valve.

stood and in the same time detailed enough to show the basic cardiac anatomy. Memorizing this basic model, the students can later upgrade their knowledge with detailed textbooks descriptions. Such useful model of the human cardiac chambers is presented here. The model proposed have not the details of atlas and textbooks images or the plastic models offered for anatomy education, but it is simple, quite correct and easily available.

In the area of congenital heart defects, the hands are basically used to show the spatial relationships of ventricular inflow, outflow and septum and thus to define the handedness (left or right) of ventricular organization during normal and abnormal conditions. ${ }^{[1]}$ Unlike this, in our model, we used the hands to show the position of the cardiac chambers and the direction of the blood running through a normal heart. Basically, this model was inspired by another educational usage of hand-finger models, as shown in the Clinically Oriented Anatomy by Moore ${ }^{[2]}$ for explanation of the superficial flexor muscles of the forearm and also the branches of intraparotid plexus.

\section{References}

1. Weinberg PM. Anatomy and classification of congenital heart disease. In: Kaiser LR, Kron IL, Spray TL, editors. Mastery of cardiothoracic surgery. 2nd ed. Philadelphia: Lippincott Williams and Wilkins; 2007. p. 639-50.

2. Moore KL. Clinically oriented anatomy. 3rd ed. Baltimore: Williams and Wilkins; 1992. p. 570, 658.

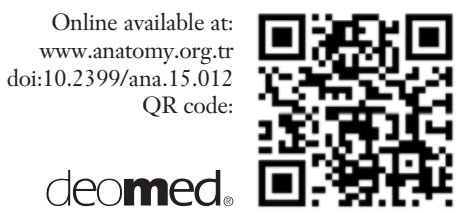

Correspondence to: Lazar Jelev, MD, PhD

Department of Anatomy, Histology and Embryology, Medical University of Sofia, blvd. Sv. Georgi Sofiisky 1, BG-1431 Sofia, Bulgaria Phone: +35929172636 e-mail: ljelev@abv.bg

Conflict of interest statement: No conflicts declared.

\footnotetext{
This is an open access article distributed under the terms of the Creative Commons Attribution-NonCommercial-NoDerivs 3.0 Unported (CC BY-NCND3.0) Licence (http://creativecommons.org/licenses/by-nc-nd/3.0/) which permits unrestricted noncommercial use, distribution, and reproduction in any medium, provided the original work is properly cited. Please cite this article as: Jelev L. Hand-finger model of the human heart - a useful educational device for understanding basic position of the cardiac chambers. Anatomy 2015;9(2):79-80.
} 